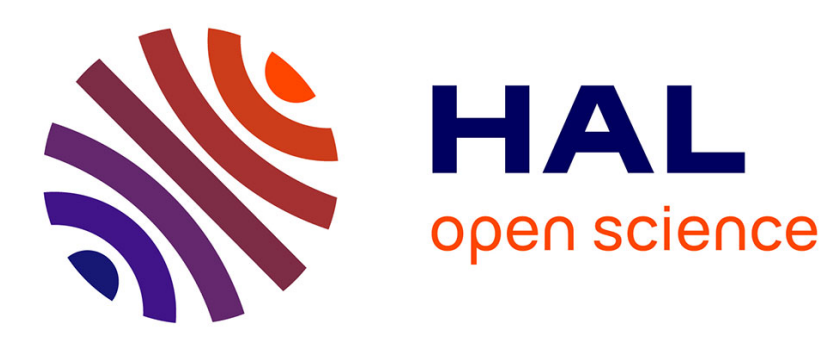

\title{
Dispersion of particles by spontaneous interparticle percolation through unconsolidated porous media
}

\author{
Franck Lominé, Luc Oger
}

\section{To cite this version:}

Franck Lominé, Luc Oger. Dispersion of particles by spontaneous interparticle percolation through unconsolidated porous media. Physical Review E: Statistical, Nonlinear, and Soft Matter Physics, 2009, 79 (5), pp.051307. 10.1103/PhysRevE.79.051307 . hal-00389904

\section{HAL Id: hal-00389904 https://hal.science/hal-00389904}

Submitted on 21 Jul 2016

HAL is a multi-disciplinary open access archive for the deposit and dissemination of scientific research documents, whether they are published or not. The documents may come from teaching and research institutions in France or abroad, or from public or private research centers.
L'archive ouverte pluridisciplinaire HAL, est destinée au dépôt et à la diffusion de documents scientifiques de niveau recherche, publiés ou non, émanant des établissements d'enseignement et de recherche français ou étrangers, des laboratoires publics ou privés. 


\title{
Dispersion of particles by spontaneous interparticle percolation through unconsolidated porous media
}

\author{
Franck Lominé* \\ Cemagref, UR ETGR, 2 rue de la Papeterie, BP 76, F-38402 St. Martin d'Hères, France
}

Luc Oger ${ }^{\dagger}$

Institut de Physique de Rennes, UMR CNRS, UR1 6251, Université de Rennes 1, 263 Avenue du Général Leclerc, 35042 Rennes Cedex, France

(Received 14 December 2008; revised manuscript received 24 February 2009; published 29 May 2009)

\begin{abstract}
We have performed extensive experimental and numerical studies of spontaneous percolation of small beads through an unconsolidated porous media made with large glass beads. In this paper, an experimental setup and a fast "discrete element method" algorithm are presented to deal with large numbers of particles during our interparticle percolation phenomenon studies. In all the experimental and numerical analyses, the size ratio between the moving beads and the stable packing was chosen larger than the geometrical trapping threshold: $\xi_{c}=\left(\frac{2}{\sqrt{3}}-1\right)^{-1}=6.464 \ldots$ We measure the longitudinal and transverse dispersion coefficients versus the height of the porous medium or the number of falling small beads. The influence of bead properties such as density, diameter, or restitution coefficients was investigated by using either steel or glass beads. The individual description of these effects and their explanations were made possible by confrontation and coupling between experimental and numerical results. Indeed, with our numerical model, individual analysis of the effects of these mechanical or geometrical parameters were made possible and carried out.
\end{abstract}

DOI: 10.1103/PhysRevE.79.051307

PACS number(s): 45.70.-n, 83.80.Fg, 81.05.Rm

\section{INTRODUCTION}

Granular materials are present in several industrial activities as well as in civil engineering and environmental phenomena. One important problem which often occurs when manipulating granular materials is spatial separation of grains according to their properties, commonly defined as segregation. This phenomenon occurs mainly when grains do not have the same size, the same shape, or the same density $[1,2]$. This segregation leads to difficulty in realizing and maintaining homogeneous mixtures of granular materials. This problem has been intensively studied [3-9] as it is the most frequently observed in industrial granular handling [2].

Let us imagine a granular material with two grain species which have very different diameters; it is obvious to observe that the smaller grains can drain totally through the piling as they are unable to obtain three stable contacts from the larger ones. So, with such diameter ratio, size segregation can simply occur under gravity, without any external mechanical action. It can be also described as a percolation problem.

In this paper, we will present results of our experimental and numerical studies of the dispersion of a group of small particles (as defined as a blob in the rest of the paper) due to the percolation process. The aim of our study is to determine which parameters are relevant for the interparticle percolation process on longitudinal and transverse dispersions of a particle blob. In all the paper, the longitudinal direction is referred to the flow direction, and the transverse one is referred to the direction perpendicular to the flow direction. We will focus on (a) the influence of the number of small par-

\footnotetext{
*franck.lomine@cemagref.fr

†luc.oger@univ-rennes1.fr
}

ticles inside a blob, (b) the size ratio between flowing particles and larger beads, (c) the different restitution coefficients (small-small or small-large), and (d) the density of the small particles. Section II will collect previous result studies and used theories and will describe the control parameters. Section III will present the experimental setup and our numerical model, then it will explain theoretical and practical approaches used in this paper. In Sec. IV, we will describe the experimental results. Finally, Sec. V, will present the numerical results and their comparison with the experimental ones.

\section{PREVIOUS STUDIES AND GEOMETRICAL CONSIDERATIONS}

The gravitational segregation approach was previously studied in simple cases [10], and also, some more complex studies have been recently released [11-13]. Nevertheless, these studies were limited to the launches of one or few small particles at the same time in order to avoid any collective interaction between small grains.

\section{A. Review of previous results}

Recent study [14] has been carried out to explore flows of small particle assemblies (or blobs) through a packing of larger spheres. This study, which was focused mainly on the influence of size ratio and the influence of blob size on the mean transit time of flowing particles, demonstrates strong modifications of flow behavior compared to the one-afterone cases $[11,12,15]$. The influence of the restitution coefficient on the particle dispersion has been also investigated in the case of isolated small beads as explained below [10]. 
Previous results $[14,16]$ have shown that the mean transit time of a set of falling particles is proportional to the height of the packing. In other words, a steady state is reached after the crossing of few grain layers and the flow of particles occurs with a constant velocity. This quasi-steady-state behavior allows the use of the classical hydrodynamic dispersion model described later on in this paper. In consequence, previous studies $[14,17]$ have shown that a mean transit time could be also determined, in a first approximation, by using

$$
C_{e}^{\star}(t)=\frac{1}{2}\left[1+\operatorname{erf}\left(\frac{V(t-\langle t\rangle)}{2 \sqrt{D_{\|}\langle t\rangle}}\right)\right],
$$

where $C_{e}^{\star}(t)$ is the elutriation profile. Equation (1) was successfully used by Bridgwater and Ingram [15] in their study of the percolation of single spheres injected one by one in a porous medium.

Ippolito et al. [12] confirmed the diffusive properties of the radial particle dispersion during flow through a packing of larger spheres. By neglecting the longitudinal dispersion, Bridgwater et al. [10] showed that the radial dispersion of the particle increases with the increase in the coefficient of restitution. This is in reasonable agreement with the tracer dispersion model of Ranz [18].

When dealing with disordered packing of spheres, it is convenient to estimate extreme values of cavity or throat sizes, which can help us to describe our experiments and their results. So, the next section points out some crucial definitions and key parameters based on geometrical considerations.

\section{B. Geometrical consideration}

First of all, in order to model this percolation process, we have chosen to use two simplifications: first, only spherical particles will be used to model the large immobile porous medium and the mobile small particles, and second, only two particle species are used during one experiment which defined one size ratio to compare moving and immobile particles. This solution is also used either experimentally or numerically. This simplification can help us to define few key geometrical parameters.

A first trivial parameter can be observed when we want to define the diameter of the largest small sphere $D_{\text {small }}$, which can pass trough any structural organization made with larger spheres of diameter $D_{\text {large }}$.

The closest and smallest organization of these spheres is the triangular one, made by three spheres mutually in contact. In this structure we can determine the diameter $D_{\text {small }}$ of the fitting small sphere, which can sit inside such a triangle formed with spheres of diameter $D_{\text {large }}$, with a critical size ratio $\xi_{c}=D_{\text {large }} / D_{\text {small }}=\left(\frac{2}{\sqrt{3}}-1\right)^{-1}=6.464 \ldots$ [19].

The second smallest and densest structure is just the extension of the previous one to the three-dimensional space: a perfect tetrahedral arrangement of four all together touching spheres. We can also obtain the size of the largest small sphere which can fit inside such a volume. Its diameter can be determined from ratio $\xi_{k}=D_{\text {large }} / D_{\text {small }}=\left(\sqrt{\frac{3}{2}}-1\right)^{-1}$ $=4.45 \ldots$. [19], but in practice such sphere is locked in the structure and our study is not concerned with this case. Nev-

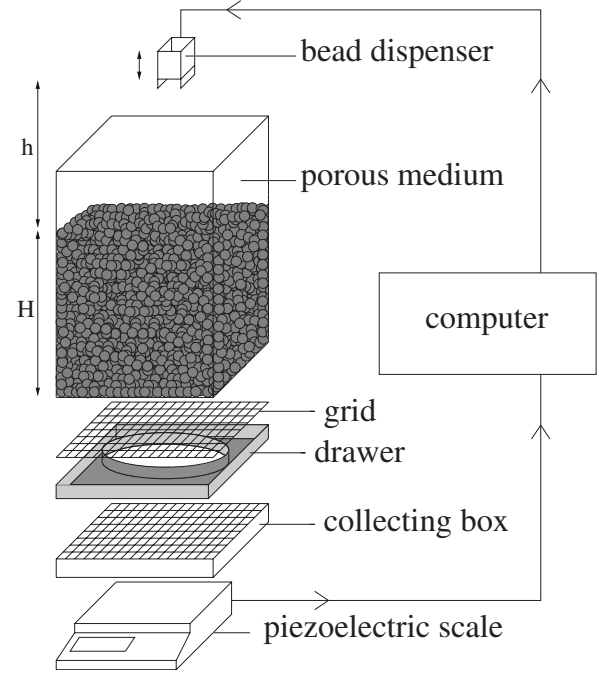

FIG. 1. Schematic description of the experimental setup. The small bead dispenser is placed at $h$ from the top of the porous media of an height $H$. The collecting box is put on the scale.

ertheless, the tetrahedral arrangement contains the smallest cavity and it can be useful to estimate the maximum amount of very small particles that can fit in it.

First, if we estimate the interstitial volume between four spheres of diameter $D$ in a tetrahedral arrangement, we obtain a volume of $\frac{D^{3}}{12}(\sqrt{2}-2 \Omega)$ as a characteristic volume of the open space, where $\Omega=3 \arccos (1 / 3)-\pi$ is the solid angle of the tetrahedron. By taking into account the well known random close packing limit $\left(\Phi_{\mathrm{RCP}}=0.64\right)[20]$ in order to have the maximum amount of small spheres, we can define $V_{p}=\Phi_{\mathrm{RCP}} \frac{D^{3}}{12}(\sqrt{2}-2 \Omega)$, as the volume occupied by the percolating particles of diameter $d$ inside the previously defined volume. So, $V_{p}$ is an estimation of the accessible volume of the smallest pore of the porous medium.

If $N$ is the number of particles of diameter $d$ injected inside the porous structure, we can now consider the ratio $N_{p}=V / V_{p}$, where $V=N \frac{4}{3} \pi\left(\frac{d}{2}\right)^{3}$ is the total volume of particles. In the rest of this paper, we will consider $N_{p}$ as one of the key parameters to characterize our results. Indeed, instead of dealing with different number $N$ of particles and diameter ratios, the parameter $N_{p}$ will let us describe the phenomena in terms of number of filled pores. By the way, it is also important to notice that $N_{p}$ is proportional to $(d / D)^{3}$.

In practice, the size distribution of the inner sphere inside these kinds of porous structures made with spheres starts at the tetrahedral value $1 / \xi_{k}$ and can reach a size up to 1 , which corresponds to an open space equivalent to the volume of the constituting large sphere $D$. The shape of this distribution is well known and always similar for disordered packings [21].

\section{EXPERIMENTAL AND NUMERICAL SETUPS WITH THEIR ANALYSIS METHODS}

\section{A. Experimental setup}

Our experimental setup, represented in Fig. 1, is made of a Plexiglas cell of $26 \times 26 \times 51 \mathrm{~cm}^{3}$ filled with a packing of 
monosize glass beads of diameter $D$. This packing, which constitutes a porous medium of height $H$, is built by random gravity deposition of the beads. This procedure gives a reproducible porosity around 0.4 [22]. Another packing of small particles of diameter $d$ is placed in a bead dispenser, which is set to a distance of $h=2.6 \mathrm{~cm}$ above the top of the porous medium. These particles are quasi-instantaneously launched on the top of the porous structure by using a vertical opening hatch. The distance $h$ is then set equal to the length of a half gate of the hatch.

As already mentioned, we will work with a ratio $\frac{D}{d}>\xi_{c}$ in all our studies. This threshold ratio, defined in Sec. II B, permits to any particle to pass freely through any pore of our porous medium. The detection system consists of two parts. First, we place under the porous medium a piezoelectric scale which records the accumulated amount of beads which succeeded to pass through the porous medium. This measurement will let us determine the time integral of the distribution of particle transit time at the exit of the porous medium. So, this analysis gives access to the mean transit time of the particles. Second, at the exit of the porous structure, we place a collecting box which divides the transverse plane into 400 square cells of $10 \mathrm{~mm}$ length side. Each square cell is keep larger than the flowing particle size in order to really measure local information (i.e., averaged over few small bead diameters). In order to let the particles exit the porous medium, the packing of larger spheres is supported by a grid with appropriate mesh size. Samson [11] showed that such grid does not influence flow properties. The number of particles in each cell is then deduced from the collected mass. These local measurements give the particles transverse position distribution. In order to really measure the transverse and longitudinal dispersions, we avoid taking into account particles that would be able to reach the walls of the Plexiglas cell. This is achieved by restricting the measured volume to a window with an horizontal circular hole of $20 \mathrm{~cm}$ diameter, which corresponds to the size of the collecting box. If particles reach the walls of the Plexiglas cell, they are trapped in the hole drawer. So, practically, we have adjusted, in each experimental set of runs, the height $H$ of the packing in order to reduce the number of trapped particles.

In all experiments, we use glass beads of diameter $D$ $=16 \mathrm{~mm}$ and mass $m_{L}=5.37 \mathrm{~g}$ to build the porous medium. Percolating particles used are steel beads with $d=1 \mathrm{~mm}$ of mass $m_{s}=4.07 \times 10^{-3} \mathrm{~g}$. Some experiments are also realized with 1 -mm-diameter glass beads of mass $m_{s}=2.1 \times 10^{-3} \mathrm{~g}$. We rebuild both packings for each experiments.

\section{B. Numerical setup and model}

Complementary to our experimental device, we have developed a numerical algorithm to fulfill our experimental study. Our program is based on "discrete element method" (DEM). In this section, we present our numerical model: the packing creation, the molecular-dynamics (MD model) approach, and the improvements made for efficiency when seeking for collision partners.

\section{Packing creation}

The first step for studying falling spheres through a porous medium is to build the large porous structure. The sim-

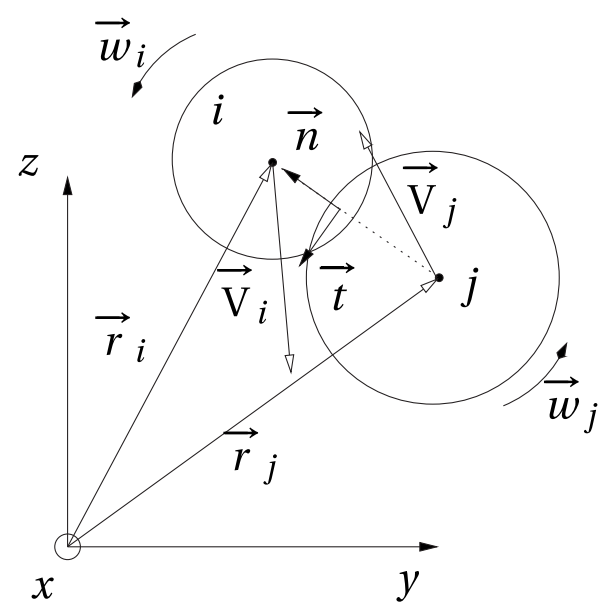

FIG. 2. Illustration of contact between two soft overlapping spheres $i$ and $j$ in the MD approach. Notations used in this paper are also mentioned.

plest and fastest porous structure is obtained by a random piling of large spheres. Several models for the construction of random packings exist; in order to build numerical stable packings with a controlled porosity; we have chosen the Powell's algorithm [23]. The key of this algorithm is to select randomly the two (for the first bottom layer) or three lower contacts from already placed spheres. This set of contacts let us determine the coordinate of the new sphere. Obviously, the new sphere positions are invalidated if there is an overlap with another already placed sphere or if its height does not fit inside a thin screening moving up layer. Then, we seek other candidates to ensure the placement.

This technique can accept also transverse periodic boundary conditions in order to avoid wall effect (i.e., local high porosity). In conclusion, this algorithm models to some extent sphere depositions under gravity, which corresponds rather well to the method used for the experimental preparation of our packings as described previously: for both the porous one made with large spheres and the blob one made with the small beads. In our simulations, the packing fraction $\Phi$ of the packing is about $60 \%$ which leads to a porosity of $40 \%$.

\section{DEM: MD model of soft spheres}

As we have achieved the creation of the two bead packings: the porous structure and the blob packing, we need to model the dynamic part of our experiments. During basic observations of the beginning of the experiments, we have seen that the moving particles present enduring contacts, which excludes the use of the "event-driven" model of the DEM. Indeed, this approach deals only with instantaneous binary collision between two particles [24,25] and can lead to spurious artifacts [26-28]. We will use a model based on "molecular dynamics of soft spheres" approach [29-31]. In the algorithm presented below, due to large size ratio between the two kinds of spheres, gravity force only acts on small particles. Figure 2 illustrates classical collision between two soft overlapping spheres $i$ and $j$ and defines notations that are used in this paper. 
MD model assumes that repulsion forces between two spheres in contact are proportional to the overlap $\delta$ between spheres $i$ and $j$ at the positions $R_{i}$ and $R_{j}$ with radii $r_{i}$ and $r_{j}$, respectively, $\delta=R_{i}+R_{j}-\left(\overrightarrow{r_{i}}-\overrightarrow{r_{j}}\right) \cdot \vec{n}$, where $\vec{n}$ is the normal unit vector. Several models of normal and tangential forces exists in the literature [29,32-36]. We have chosen to use the widely sprayed "linear spring dashpot" (LSD) model [30,37-39] for the normal force and the Cundall and Strack [29] model for the tangential force. With these models, normal and tangential forces are modeled, respectively, by

$$
\begin{gathered}
F_{n_{j i}}=k_{n} \delta+\gamma_{n} \dot{\delta}, \\
F_{t_{j i}}=-\psi \min \left(\left|k_{t} \varphi\right|, \mu \| F_{n_{i j}} \mid\right),
\end{gathered}
$$

where $k_{n}$ is a stiffness coefficient, $\dot{\delta}$ is the time derivative of $\delta$, and $\gamma_{n}$ is a damping constant. $\varphi$ is the tangential overlap defined by

$$
\varphi=\int_{t_{\alpha}}^{t_{\beta}} \vec{t} \cdot \vec{d} l,
$$

where $\mu$ is the friction coefficient and $t_{\alpha}$ and $t_{\beta}$ are the two overlap limits. $k_{t}$ is a tangential stiffness coefficient which is assigned, in order to ensure a correct energy restitution, to a value of

$$
k_{t}=\frac{2}{7}\left(\frac{\pi^{2}+\left(\ln e_{t}\right)^{2}}{\pi^{2}+\left(\ln e_{n}\right)^{2}}\right) k_{n},
$$

where $e_{n}$ and $e_{t}$ are normal and tangential energy restitution coefficients. $\vec{t}$ is the unit tangent vector. The symbol $\psi$ takes the values \pm 1 according to the sign of tangential relative velocity. Two kinds of collisions are involved in our experiments. The first one is a collision between a small moving bead and a large immobile one, and the second one is a collision between two percolating moving particles. The restitution coefficients of these two kinds of collisions will be, respectively, denoted $e_{1}$ and $e_{2}$ in the rest of this paper.

In order to integrate the equations of motion for the determination of the positions and velocities at time $t+d t$, we need to adapt the time step $d t$. Generally, $d t$ is taken to be equal to $d t=\min \left(t_{c}\right) / 100$, where $t_{c}$ is the contact duration between $i$ and $j$ at time $t$. With the LSD model, the contact duration $\tau_{n}$ (defined by the existence of an overlap), in normal direction is $\tau_{n}=\pi\left[\frac{k_{n}}{m_{i j}}-\left(\frac{\gamma_{n}}{2 m_{i j}}\right)^{2}\right]^{1 / 2}$. If Eq. (5) is satisfied, we have $\tau_{n}=\tau_{t}$, where $\tau_{t}$ is the contact duration in tangential direction, and then we have $t_{c}=\tau_{n}$. The time integration is performed using a Verlet's algorithm [40]. In spite of the periodicity of the porous medium generated with the Powell's algorithm, interactions between moving spheres do not take into account these periodicity (i.e., only small sphere in the same periodic space can collide).

\section{Algorithm efficiency}

The computation time is controlled by two main loops: one over the number of moving spheres and another one for the determination of the neighbors for each sphere. As a classical DEM simulation, the system is divided in cells in order to reduce the number of possible available neighbors which have to be tested by the program. For each grid of cells, a linked list is associated. These linked lists permit us to easily identify particles that are in the same or adjacent cells and avoid looping over all particles. Indeed, in the linked-list method, each sphere links to another sphere which is in the same cell [41]. But this method needs to have small number of particles in each cell to be efficient. For that, a common method is to set the cell size smaller as possible but in the order of few large sphere diameters $D$. During our spontaneous percolation experiment, the particles of diameter $d$ are very small compared to this cell size. So despite the previous cell size optimization, the number of small particles can be very important in each cell, and by consequence this cell method losses partially its efficiency.

In order to remedy to this problem, our first improvement is to use two cell grids for achieving desired performances: a large grid for the spheres building up the porous media and a smaller one for the moving particles. This permits us to reduce the number of small particles in the corresponding grid. By consequence the search of possible overlaps is divided into two steps: first, we look for contacts between two small particles within the associated grid and linked list; then, we look for contacts between a moving particle and a sphere of the porous medium with the other corresponding grid and linked list.

In the linked-list method, it is necessary to adjust the links between particles that are localized in the same cells when the particles have moved in or out of a given cell. As only small particles can move, only one linked list is concerned. But as the number of cell can be very important, the full reinitialization of this list, at each time step, needs too much time. To avoid this time consumption, we have implemented a doubly linked-list [16] which constitutes a great improvement compared to the traditional linked-list method. In our method, each sphere links to another sphere which is in the same cell and each linked sphere has also a reverse link (to identify the sphere which makes a link to it).

With this method, it is easier to update the linked list and the list of spheres contained in each cell without global reinitialization. When a particle moves in or out of a cell, only links and reverse links of this sphere and the ones of linked and reversely linked spheres have to be changed.

The third improvement used in our simulation was to make a parallel version of our code with message passing interface (MPI) coding in order to ensure good performances for a relative high number of particles. Indeed, each moving sphere calculation is limited to their neighbors so it is very easy to divide the global calculi in a subset of small number of spheres calculated by each MPI processes.

Most calculations were performed on 64 bit architectures at $1.8 \mathrm{GHz}$. For a duration example, a simulation of $N$ $=1000$ particles in a packing with $H / D=14$ and size ratio $D / d=10$ running on a single processor takes approximately 10 and $5 \mathrm{~h}$ if using two processors. The same simulation with $D / d=6.5$ can take twice. When the number of falling particles increases, simulation can take a very long time. For example, with $N=10000$ or more, the simulation can run during two or three weeks on a single processor. Thanks to parallelization, this time can be divided by the number of processors. 


\section{Theoretical and practical approaches}

The two previous sections, describes, respectively, our experimental setup and the numerical program used to perform the study of interparticle percolation of small particles through an unconsolidated porous medium. In classical hydrodynamics dispersion process, few quantities are generally measured or observed. Depending of the ability of inner measurements or not, we can obtain local or global values of some physical quantities. With the experimental device presented previously, it is only possible to determine the mean transit time of the particle blob and particle dispersions. But depending on the setup considered (experimental or numerical one), the way to determine these quantities can be different. The aim of this section is to define our measurement protocol and to review accessible quantities for these two setups.

\section{Determination of experimental dispersion coefficients}

Our experimental device does not permit us to follow trajectories of particles inside the porous structure. Nevertheless, the collecting box placed under the packing of the larger spheres was built in order to investigate the transverse dispersion of particles. The distribution function of the particle exit positions requires counting the number of particles in each cell of the collecting box. This is done by weighting of the mass contained in each cell of the collecting box. We have to point out that these measurements need a quite long time compared to the experiment duration. If we launch a blob of particles, each cells $i$ of the collecting box placed under the porous medium have a mass $m_{i}\left(x_{i}, y_{i}\right)$ of particles, and we can define the distribution particle function $p_{i}\left(x_{i}, y_{i}\right)$ by

$$
p_{i}\left(x_{i}, y_{i}\right)=p_{i}=\frac{m_{i}\left(x_{i}, y_{i}\right)}{\sum_{i} m_{i}\left(x_{i}, y_{i}\right)},
$$

where $x_{i}$ and $y_{i}$ are positions of the center of the cell $i$ in the plane perpendicular to the flow direction.

We can easily assume that the transverse distribution function is roughly homogeneous and reproduces the horizontal isotropic properties of the porous medium. In consequence, it is convenient to define the particle position distribution function only in one dimension $r$ without an azimuthal component. For each particle, we have $r_{i}^{2}=x_{i}^{2}+y_{i}^{2}$.

So, the variance $\left\langle(\Delta r)^{2}\right\rangle$ of the position distribution is related to the transverse dispersion coefficient $D_{\perp}$ by

$$
\left\langle(\Delta r)^{2}\right\rangle=\sum_{i} p_{i}\left(r_{i}-\langle r\rangle\right)^{2}=4 D_{\perp} t .
$$

As we have already mentioned in Sec. II A, Lominé and Oger [14] put in evidence the existence of stationary regime with constant velocity $V$ during flow of particles. If we define a mean transit time by

$$
\langle t\rangle=\frac{H}{V} .
$$

Then, Eq. (7) can be written as

$$
\left\langle(\Delta R)^{2}\right\rangle=\sum_{i} p_{i}\left(R_{i}-\langle R\rangle\right)^{2}=4 D_{\perp} \frac{H}{V},
$$

where $R$ is the position of particles at the output of the porous structure. So Eq. (9) let us determine the transverse dispersion coefficient $D_{\downarrow}$.

The main phenomenon, which occurs inside the porous space, is longitudinal and transverse dispersions that is classically described using an advection-dispersion equation (ADE) approach. With this consideration, the concentration $C(r, t)$ of particles follows the equation $[42,43]$

$$
\frac{\partial C(r, t)}{\partial t}+U \nabla C(r, t)=D_{\|} \frac{\partial^{2} C(r, t)}{\partial r_{\|}^{2}}+D_{\perp} \frac{\partial^{2} C(r, t)}{\partial r_{\perp}^{2}},
$$

where $C(r, t)$ is the particle concentration at position $r$ at time $t, U$ is the interstitial velocity of the flow, and $D_{\|}$and $D_{\perp}$ are, respectively, the longitudinal and transverse dispersion coefficients. It should be noted here that Eq. (10) is a continuum approximation of a discrete phenomenon. Van Genuchten et al. $[44,45]$ showed that if we consider Eq. (10), in one dimension $z$, an analytical solution for a semi-infinite system and for a continuous injection $\left[C(0, t>0)=C_{0}\right]$, with initial conditions $C(z, 0)=0$ and $\frac{\partial C(\infty, t)}{\partial t}=0$, is

$$
C_{e}(z, t)=\frac{C_{0}}{2}\left[\exp \left(\frac{z V}{D_{\|}}\right) \operatorname{erfc}\left(\frac{z+V t}{\sqrt{4 D_{\|} t}}\right)+\operatorname{erfc}\left(\frac{z-V t}{\sqrt{4 D_{\|} t}}\right)\right],
$$

where $V$ is the mean velocity.

A solution of Eq. (10) for an instantaneous injection, which is our case studies, can be obtained by the differentiation with respect to time of Eq. (11). Therefore, Eq. (11) corresponds to the time integration of the transit time distribution for an instantaneous injection. Despite the simplicity of the model, Eq. (11) let us determine the dispersion of particles in the flow direction. As we will see in Sec. IV A, the adjustments of transit time distributions at the exit of the porous medium are well fitted with Eq. (11), and more sophisticated models will not help to improve accuracy of these adjustments. Then, it is possible to access the longitudinal dispersion coefficient $D_{\|}$by considering the time integration profile of particle transit time distribution and Eq. (11). Equation (11) could also be used to determine the velocity $V$ or the mean transit time via Eq. (8), which could be also determined with Eq. (1). The observed differences of the mean transit times determined with these methods will be discussed in Sec. IV A.

\section{Determination of numerical dispersion coefficients}

Numerically, it is possible to access individual particle positions, anywhere at any time, inside the packing of larger spheres. Then, contrary to experimental case, variance can be evaluated at every time during the grains fall. If we note $r_{k}$, the position of a particle $k$ in the horizontal plane in such way that $r_{k}^{2}=x_{k}^{2}+y_{k}^{2}$, where $x_{k}$ and $y_{k}$ are the particle positions, we can deduce the position fluctuations. So the variance of the position distributions of the $N$ moving particles in this plane is 


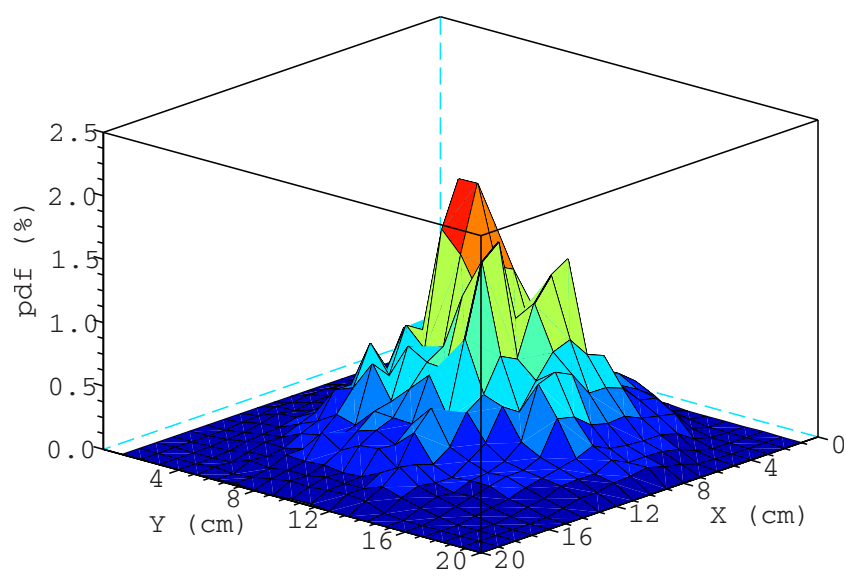

FIG. 3. (Color online) Distribution function of particle positions at the exit of the porous medium for a launch of 10000 glass particles of mass $m_{s}=2.1 \times 10^{-3} \mathrm{~g}$ and with a porous medium of height $H / D=8.125$.

$$
\left\langle(\Delta r)^{2}\right\rangle=\frac{1}{N} \sum_{k=1}^{N}\left(r_{k}-\langle r\rangle\right)^{2},
$$

where $\langle r\rangle=\frac{1}{N} \sum_{k=1}^{N} r_{k}$. In the same manner, if the particle position in the flow direction is denoted by $z_{k}$, we have

$$
\left\langle(\Delta z)^{2}\right\rangle=\frac{1}{N} \sum_{k=1}^{N}\left(z_{k}-\langle z\rangle\right)^{2},
$$

with $\langle z\rangle=\frac{1}{N} \sum_{k=1}^{N} z_{k}$.

Then, in numerical case, the two dispersion coefficients $D_{\perp}$ and $D_{\|}$will be deduced from time evolution of $\left\langle(\Delta r)^{2}\right\rangle$ and $\left\langle(\Delta z)^{2}\right\rangle$ with classical relations

$$
\left\langle(\Delta r)^{2}\right\rangle=4 D_{\perp} t \text { and }\left\langle(\Delta z)^{2}\right\rangle=2 D_{\|} t .
$$

\section{EXPERIMENTAL STUDY}

With the experimental setup presented previously in Sec. III A, we have performed experiments to determine dispersion coefficients. When a blob of $N$ particles is free to fall on the surface of the packing made of larger spheres, particles flow through the porous medium and disperse due to collisions with the porous matrix.

\section{A. Transverse dispersion}

Experimentally, it is possible to access particle dispersion at the exit of the porous medium. For example, Fig. 3 illustrates the particle distribution function for a launch of 10000 glass particles.

To determine transverse dispersion coefficient $D_{\perp}$ with Eq. (9), it is first required to measure $V$, the mean velocity which is related to $H /\langle t\rangle$, where $\langle t\rangle$ is the mean transit time. For that purpose, we have done several launches of particles for various number $N$ and various heights $H$ of the packing. Using Eq. (11), it is possible to fit the time integral profile of the time transit distribution gathered by the piezoelectric scale. Figure 4 shows the evolution of particle mass collected

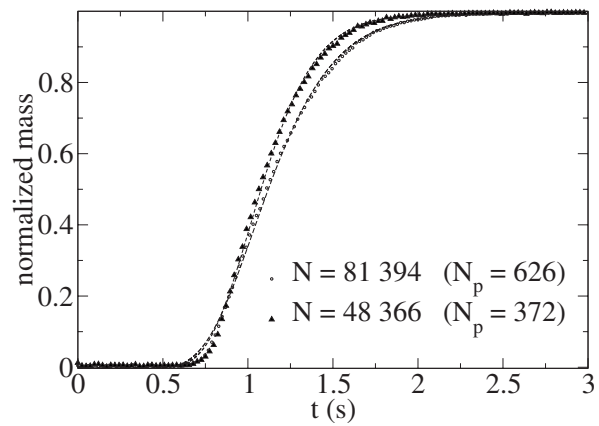

FIG. 4. Normalized mass measurements delivered by the piezoelectric scale. A fit with Eq. (11), and determined with unweighted least-squares method, is also represented with continuous lines. $N$ corresponds to the number of falling steel particles and $N_{p}$ is the rescaled value, which was defined in Sec. II. Here $H / D=10$.

under the packing of larger spheres, with respect to time for two values of $N$. The values of corresponding $N_{p}$ are also indicated. We have drawn also the best fits of the experimental profiles using Eq. (11).

In spite of the simple hydrodynamic model used here [Eq. (10)], the agreements between Eq. (11) and experimental data, show that the time integral profile of the transit time distribution is very well fitted by this equation. These adjustments where done by using unweighted least-squares fitting method and letting all parameters, in Eq. (11), free to adjust. The value of $z$ at the end of adjustment was found to match the $H$ values in all cases. Nevertheless, adjustments were also done by fixing $z$ to $H$ and letting all other parameters free to being adjust. In this case, final values of $V$ and $D_{\|}$ were found to be very closed to the values obtained without fixing the $z$ parameter. Moreover, more sophisticated advection-dispersion models [including retardation factor $\left(R_{e}\right)$, general first-order deposit factor $(K)$, and an injection source $(\gamma)][44,45]$ were investigated without giving noticeable differences compared to the use of Eq. (11). Indeed, small differences on values $V$ and $D_{\|}$observed with these models were found to stay in incertitude between two distinct experiments. The general form of the advection dispersion equation which was also tested is

$$
R_{e} \frac{\partial C(z, t)}{\partial t}-D_{\|} \frac{\partial^{2} C(z, t)}{\partial z^{2}}+V \frac{\partial C(z, t)}{\partial z}+K C(z, t)+\gamma=0 .
$$

Nevertheless, Eq. (11) remains the simplest and best adjustment law for fitting elutriation profile of our experiments. Moreover, in order to complete this already good accuracy and to have better reliable data, each experimental data point presented in this paper is a statistical mean of at least ten fully independent experiments (i.e., newly built large and small packings). We also present incertitude determined with largest fluctuations between experiments. Incertitudes due to all hydrodynamical models used are also incorporated.

Figure 5 shows evolution of mean transit time with $H / D$. Linear fits, determined with unweighted least-squares method, are also represented. Results have been obtained for different particle blobs. As already seen in [14], the mean 


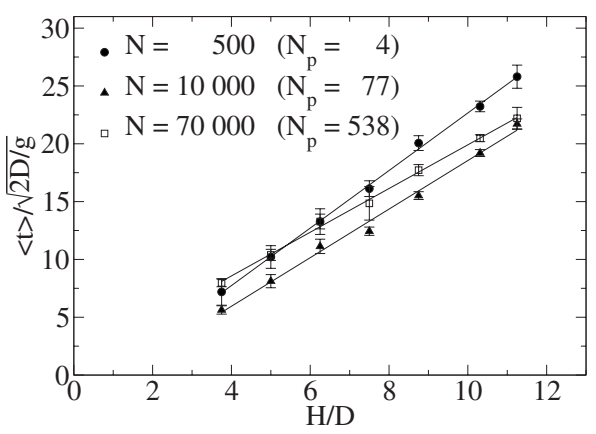

FIG. 5. Mean transit time dependency on $H / D$ for $N=500, N$ $=10000$, and $N=70000$. Results obtained with steel percolating particles of $d=1 \mathrm{~mm}$ inside glass beads of diameter $D=16 \mathrm{~mm}$. Linear fits are also represented.

flow velocity depends on the number of particles that travel inside the porous space. From the slope of the mean transit time versus the height of the packing, we can deduce the mean flow velocity $V$ of the falling particles.

Using the first part of Eq. (9), we have determined the variance of transverse particle distributions at the output of the porous medium versus the packing height of the larger spheres. Figure 6 shows the results obtained for different sets $N$ of small steel and glass spheres of $d=1 \mathrm{~mm}$ diameter. We can observe that $\left\langle(\Delta R)^{2}\right\rangle$ is proportional to the height $H$ of the porous medium and then follows the second part of Eq. (9).

Figure 7 shows the transverse dispersion coefficients, determined by Eq. (9) for the different set sizes of the small particles versus the scale factor $N_{p}$. Two kinds of particles were studied: steel or glass beads. This curve shows clearly that the transverse dispersion increases with the number of particles flowing through the packing of larger spheres. The larger particle blobs are, the bigger is the "dense kernel" of the particle cloud. So, near the surface of this "kernel," the ejections of the moving particles toward less dense regions is facilitated. The increase in that surface versus $N_{p}$ and, by consequence with the number of particles $N$, can explain the results observed in Fig. 7. Indeed as observed by Lominé and Oger [14], an individual particle, similar to the one on the cloud periphery, has a higher transit time due to a more important transverse exploration of the porous medium, com-
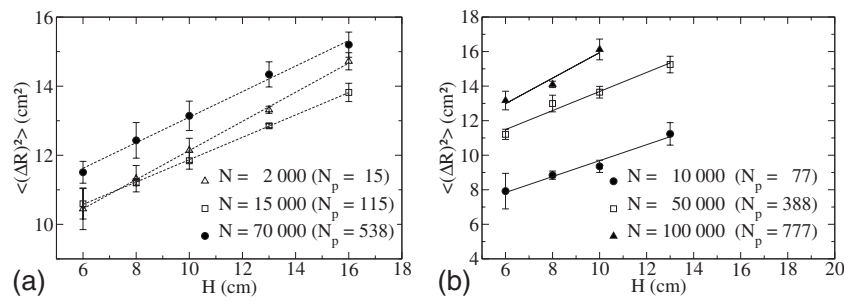

FIG. 6. Variance of the particle distribution function in the transverse direction at the output of the porous medium versus $H$ for different numbers of falling particles. In these experiments, we used small steel beads (a) and glass ones (b) of diameter $d=1 \mathrm{~mm}$ inside packings of glass beads of diameter $D=16 \mathrm{~mm}$ (i.e., size ratio $D / d=16)$. Unweighted linear least-squares regressions are also represented.

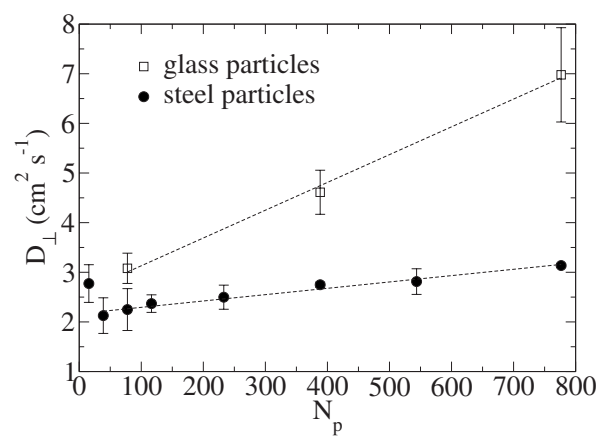

FIG. 7. Evolution of transverse dispersion coefficient $D_{\perp}$ versus $N_{p}$. The experiments were performed with small steel $(\bullet)$ and glass ( $\square$ ) beads of diameter $d=1 \mathrm{~mm}$ through a porous medium made with glass bead of diameter $D=16 \mathrm{~mm}$ (size ratio $D / d=16$ ). We have also represented unweighted linear least-squares regressions.

pared to a particle with an important neighborhood. This phenomenon results in an higher dispersion coefficient.

Despite the fact that only few points of measurements were present for glass beads, it is possible to notice that the values of transverse dispersion coefficients for the small glass beads are higher than for the steel ones. At this stage, it is not possible to explain truly the reasons of these large values, however we can only express several possibilities, as the change in the moving bead properties results in modifications of many physical and mechanical parameters. The modification of surface properties is our first assumption and can produce different friction coefficients. This assumption is possible but seems not so efficient as the flow of particles is essentially made by ballistic movements and instantaneous collisions. The second assumption is more evident and can be expressed as the change in the particle density (from $7.8 \mathrm{~g} \mathrm{~cm}^{-3}$ down to $2.5 \mathrm{~g} \mathrm{~cm}^{-3}$ for steel and glass beads, respectively). So, glass particles are less subject to gravity force due to their lower density. This generates a smaller acceleration force during the falling trajectories between two consecutive layers and, by consequence, changes the ability to explore laterally the porous space. The last possibility which can explain this increase in the transverse dispersion coefficient is the change in contact properties and more precisely the restitution coefficient with the spheres of porous medium or between two falling particles. This assumption seems crucial for our studies; but in practice, it is impossible to change this restitution coefficient without changing also some other properties of the beads. This is the reason why the influence of energy dissipation will be one of the main key factor studied later, through our numerical simulations. In the same manner, we will analyze also the effects of the density of the percolating particles independently of the other parameters.

\section{B. Longitudinal dispersion}

We have already mentioned that, with the use of Eq. (11), it is possible to determine the longitudinal dispersion coefficient by fitting the time integration profile of transit times, obtained at the exit of the porous medium. For the small steel beads falling through a packing of larger glass spheres, we 


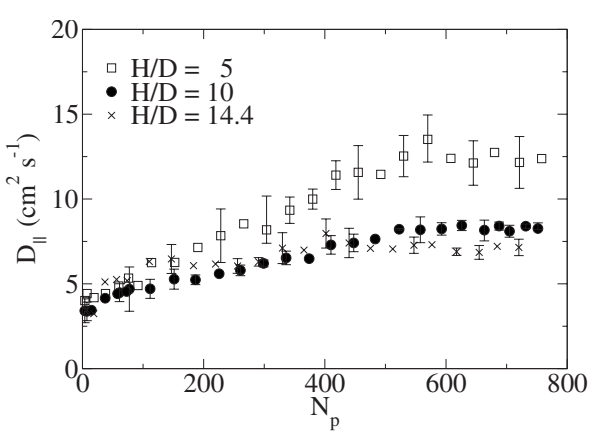

FIG. 8. Dispersion coefficients in the flow direction determined with Eq. (11), versus the parameter $N_{p}$ for three different packing heights $H$. In these experiments, we use small steel beads of diameter $d=1 \mathrm{~mm}$ inside a packing of glass beads of diameter $D$ $=16 \mathrm{~mm}$ ( size ratio $D / d=16$ ). In order to improve visual quality of the figure, all error bars are not displayed.

have determined the longitudinal dispersion coefficients versus the different sizes of particle blobs. Figure 8 shows evolutions of these coefficients with the parameter $N_{p}$, for three heights $H$. We can observe that the dispersion coefficient increases with $N_{p}$ (i.e., the number of particles $N$ ). When $N_{p}$ is larger, more neighboring pores are occupied by the moving beads at the same time. This situation can be translated as a smaller probability for the moving particles to find rapidly a possible vertical path, which results in a larger dispersion of particles in the longitudinal direction. Figure 8 shows also that the dispersion coefficient measured for $H / D=5$ differs from those measured for $H / D=10$ and for $H / D=14$.4. This difference demonstrates that an intermediate state in the top layers of the porous medium (around $H / D=5$ ), is not negligible for the study of the rest of the flow. This different behavior is due to the particle rebounds on the surface of the porous medium (recall that the dispenser is $h=2.6 \mathrm{~cm}$ above the top layer of the porous medium) and also due to the transition phase which occurs in the first few layers. This second effect can be described as an equilibrium zone where the falling particles collide rapidly with each other and with the first layers of large spheres.

\section{NUMERICAL STUDY}

As mentioned previously, some parameters are experimentally impossible to modify independently of other ones. This is the reason why numerical simulations of our experiments is useful to complete our analysis of the interparticle percolation phenomenon.

We present the numerical results of our simulations, obtained with the program presented previously in Sec. III B, of the flow of a blob of small particles through a porous medium. As illustrated by the series of snapshots presented in Fig. 9, numerical simulations let us have also access to positions of particles inside the porous space. In order to be as close as possible to real experiments, density of small particles $\rho_{s}$ has been taken equal to $7.8 \mathrm{~g} \mathrm{~cm}^{-3}$, and we choose a value of $2.5 \mathrm{~g} \mathrm{~cm}^{-3}$ for the density $\rho_{l}$ of the glass spheres. Moreover, as the experimental setup, we rebuild each packings for each simulation. Each numerical data

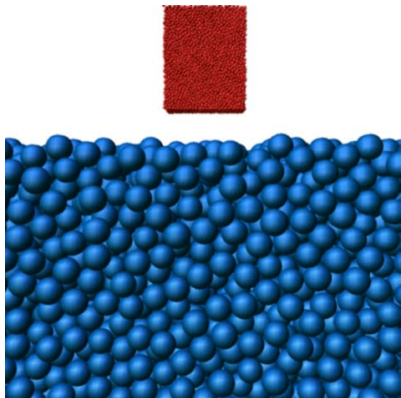

(a)

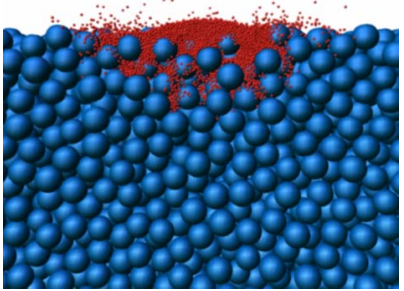

(c)

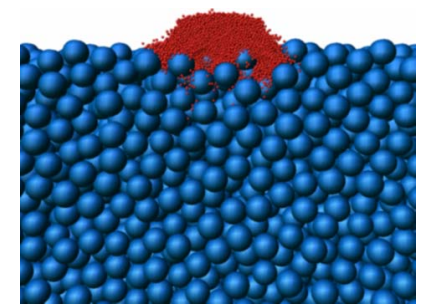

(b)

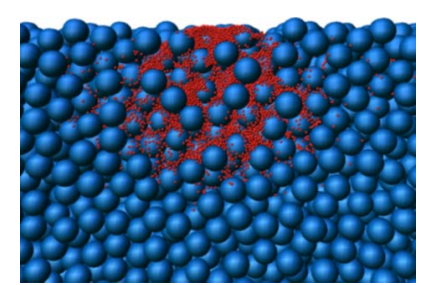

(d)
FIG. 9. (Color online) Snapshots of flow of 20000 particles through a porous medium. Note that for convenience only half system is represented.

which are presented in this paper are coming from a statistical mean of several simulations. Error bars can be deduced from these replicate simulations.

\section{A. A diffusive process}

Figure 10 presents variances, $\left\langle(\Delta r)^{2}\right\rangle$ and $\left\langle(\Delta z)^{2}\right\rangle$ calculated with Eqs. (12) and (13), of particle position distribution for $N=8000$ versus time. In this figure, the linear fits obtained by the unweighted least-squares method are also represented. We can notice that this linear evolution of the two variances with time is a typical proof of a diffusive property. The deviation from the linear regression observed for larger

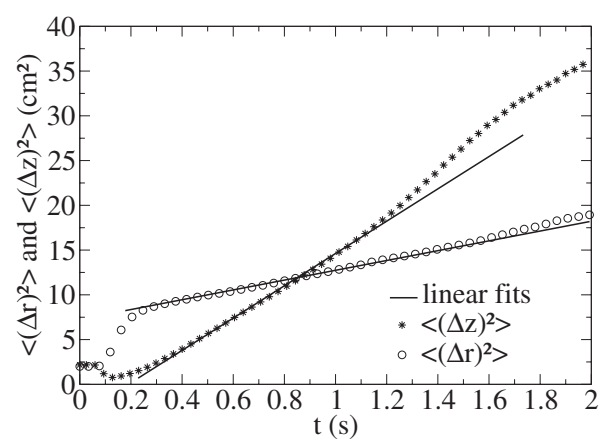

FIG. 10. Time evolutions of $\left\langle(\Delta z)^{2}\right\rangle$ and $\left\langle(\Delta r)^{2}\right\rangle$ versus time obtained by the MD model of soft spheres, with $N=8000, D / d$ $=10, D=20 \mathrm{~mm}, e_{1}=0.84, e_{2}=0.99$, and $H / D=14$. Linear fits obtained with unweighted least-squares method are represented. The linear evolution of $\left\langle(\Delta z)^{2}\right\rangle$ is observed between 0.2 and $1 \mathrm{~s}$ as shown by the straight line. After $1 \mathrm{~s}$ the decrease in the moving particles amount explains the divergence which is only an artifact of the calculus method. The same behavior is observed on evolution of $\left\langle(\Delta r)^{2}\right\rangle$ but divergence starts to be visible after $1.5 \mathrm{~s}$. 


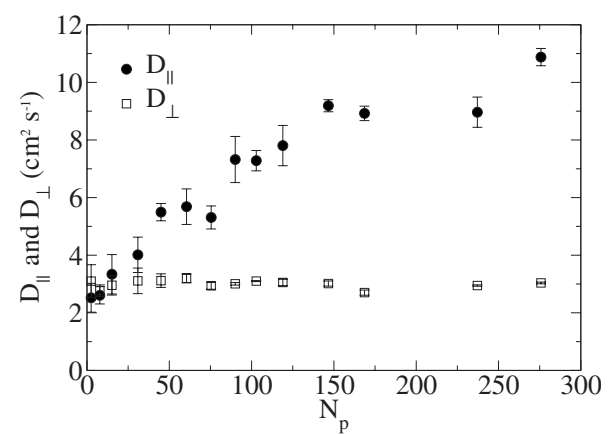

FIG. 11. Evolution of dispersion coefficients versus $N_{p}$ for a size ratio $D / d=10$. These results have been obtained with $H / D=14$, $D=20 \mathrm{~mm}, e_{1}=0.84$, and $e_{2}=0.99$. Error bars are also represented.

values of $t$ is mainly due to the finite size of our simulations. Indeed, at long time, some particles have already reached the bottom of the porous medium which implies that the calculus of $\left\langle(\Delta r)^{2}\right\rangle$ and $\left\langle(\Delta z)^{2}\right\rangle$ are less and less accurate. In the other limit, for the small values of $t$, we can also observe a deviation from the fits in Fig. 10 which can be explained by the time needed to reach a diffusive behavior and already mentioned in Sec. IV B for small $H / D$. Diffusive motion of an isolated falling particle was observed by Ippolito et al. [12], and our simulations prove the same behavior for a blob of particles. This fact confirms our assumption on the diffusive behavior mentioned for our experimental results.

\section{B. Effect of blob size $N_{p}$}

In the previous section, we have demonstrated that we can deduce the dispersion coefficients from our numerical simulations. So right now, we can analyze the coefficients $D_{\|}$and $D_{\perp}$ for various numbers of injected particles, expressed through the parameter $N_{p}$, into the packing of large spheres. As we have previously seen in Sec. II B, $N_{p}$ will let us describe influence of $N$ in terms of number of filled pores. Figure 11 shows these two coefficients versus $N_{p}$ for experiments with $D / d=10$ and $H / D=14$.

Figure 11 reveals that the transverse dispersion coefficient $D_{\perp}$ does not truly depend on the number of injected particles. On the other hand, we can notice that the dispersion coefficient $D_{\|}$in the flow direction increases with the number of particles. In order to compare with our experimental results (size ratio $D / d=16$ ) we have also represented the behavior of these two dispersion coefficients in this case (see Fig. 12).

Unlike the results presented in Fig. 11 and despite relatively large incertitudes in Fig. 12, we can notice that the transverse dispersion coefficient $D_{\perp}$ increases with $N_{p}$. This evolution is in good agreement with the experimental results presented in Fig. 7. By making a comparison between Figs. 11 and 12 , we can also notice that $D_{\|}$for $N_{p} \approx 50$ is smaller when $D / d=16$ than the one for $D / d=10$. So, it is obvious that the particle dispersion depends on size ratio $D / d$. Then the next section will present the ratio $D / d$ influence.

\section{Effect of size ratio $D / d$}

Dependency of $D_{\perp}$ on size ratio $D / d$, observed between Figs. 11 and 12, is confirmed in Fig. 13 which represents the

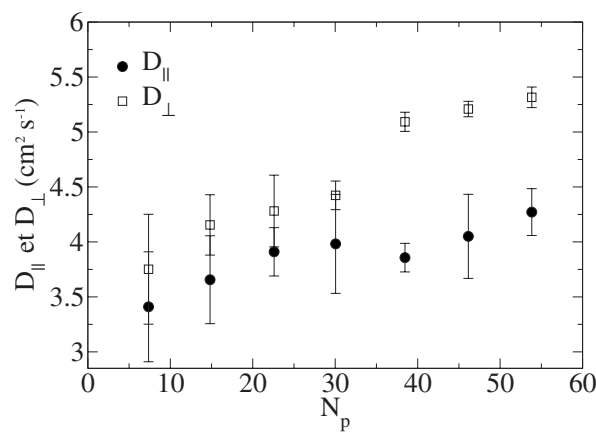

FIG. 12. Evolution of dispersion coefficients versus $N_{p}$ for a size ratio $D / d=16$. Error bars are shown for accuracy analysis. Results are obtained with $H / D=14, D=20 \mathrm{~mm}, e_{1}=0.84$, and $e_{2}$ $=0.99$.

evolution of $D_{\perp}$ with $D / d$ for different particle numbers $N$.

This figure demonstrates that, when the size ratio is lower than $12, D_{\perp}$ increases with $D / d$. Incertitudes between simulations does not allow us to determine a significant dependency of $D_{\perp}$ on $N$ for $D / d<12$, which is in agreement with results of Fig. 11. The smaller the particles are, the more they can laterally move along long distances. When size ratio is higher than 12, we can notice that the global behavior of $D_{\perp}$ is nearly constant around $D_{\perp}=4 \mathrm{~cm}^{2} \mathrm{~s}^{-1}$, but we can see a larger fluctuation of $D_{\perp}$ with $N$. This is comparable to Fig. 12.

In the same way, we have represented in Fig. 14 the evolution of longitudinal dispersion coefficient $D_{\|}$versus the size ratio $D / d$. When the size ratio is small $(D / d<9)$, particles have difficulties to pass trough the packing of larger spheres, and this fact results in large fluctuation of the dispersion coefficient in the flow direction. Indeed for such size ratio, the particle blob needs more time to reach the steady state with constant velocity and determination of the slope of $\left\langle(\Delta z)^{2}\right\rangle=f(t)$ is less accurate than for $D / d>9$ cases.

We can observe that the longitudinal dispersion coefficient $D_{\|}$fluctuates in opposition to the transverse one: $D_{\|}$ decreases when the size ratio increases up to the ratio $D / d$ $=12$, then remains constant as $D_{\perp}$ when $D / d$ is larger. These two evolutions can be easily explained as a basic transfer of movements between the two kinds of displacements: when $D / d$ is small, the particles have a smaller probability of mov-

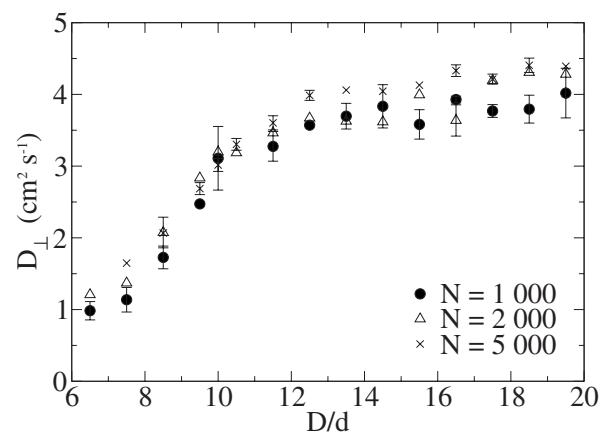

FIG. 13. Evolution of $D_{\perp}$ versus $D / d$ for different values of $N$. The results are obtained with $H / D=14, D=20 \mathrm{~mm}, e_{1}=0.84$, and $e_{2}=0.99$. To improve the readability, only significant error bars are displayed. 


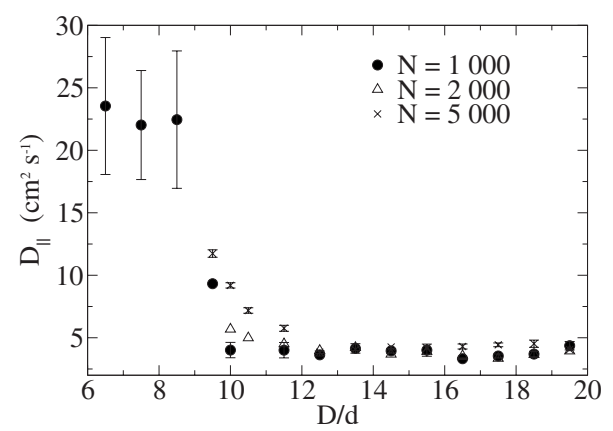

FIG. 14. Evolution of $D_{\|}$versus $D / d$ for different values of $N$. Results have been obtained by the MD model of soft spheres with $H / D=14, D=20 \mathrm{~mm}, e_{1}=0.84$, and $e_{2}=0.99$.

ing outside a pore in the horizontal direction and thus a greater probability of passing through the vertical pore. By opposition, if they can move freely laterally (i.e., inside or outside horizontal pores) they have lower probabilities to have larger fluctuations in the vertical directions.

\section{Effect of the restitution coefficients $e_{1}$ and $e_{2}$}

We have performed a series of simulations to study the influence of the two different energy restitution coefficients (small-small or small-large) on particle dispersion. Figure 15(a) shows the transverse dispersion coefficients $D_{\perp}$ for different particle blob sizes. The results show that $D_{\perp}$ is almost independent of the restitution coefficient $e_{1}$, where $e_{1}$ is the coefficient for a collision between a moving particle and a sphere of the porous medium.

In the same manner, the evolution of the longitudinal dispersion coefficient $D_{\|}$versus $e_{1}$ is illustrated in Fig. 15(b). We can summarize these evolutions by noticing that the dispersion coefficient in the flow direction decreases when collisions between the porous medium and percolating particles are less dissipative. This can be easily explained by considering a single particle falling down toward a pore. The particle trajectory must be aligned with the pore hole to pass through it without bouncing around. If not, the larger the restitution coefficient is, the more important the rebounds of this particles are. In such a case, longitudinal crossing of individual pore is more difficult and leads to a decrease in the longitudinal dispersion coefficient.

Now, we analyze the influence of the restitution coefficient $e_{2}$ between two moving particles on the particle disper-
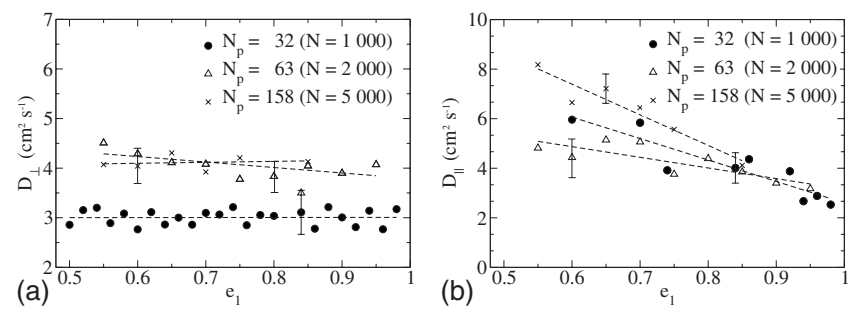

FIG. 15. Evolution of (a) $D_{\perp}$ and (b) $D_{\|}$with $e_{1}$ for $N=1000$, $N=2000$, and $N=5000$. The results are obtained with $H / D=14$, $D / d=10, D=20 \mathrm{~mm}$, and $e_{2}=0.99$. Unweighted linear leastsquares regressions are also represented.
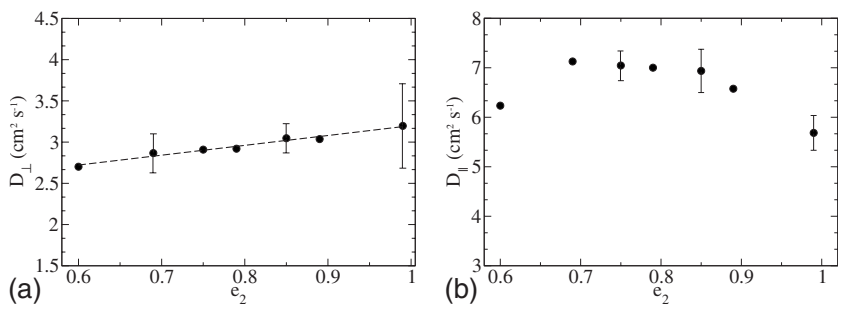

FIG. 16. Evolution of (a) $D_{\perp}$ and (b) $D_{\|}$versus $e_{2}$ for $N$ $=2000, D / d=10, D=20 \mathrm{~mm}, H / D=14$, and $e_{1}=0.84$. An unweighted linear least-squares regression is represented in case (a).

sion. Figure 16(a) shows the variation in the transverse dispersion coefficient $D_{\perp}$ versus the coefficient of restitution $e_{2}$. We could notice that the larger the coefficient of restitution $e_{2}$ is, the more the particles disperse laterally. But, due to relatively large error bars, it not possible to confirm strongly this increase in $D_{\perp}$ with $e_{2}$. It could be interesting to perform a set of simulations for larger values of $N$.

On the other hand, if we consider Fig. 16(b), which represents the evolution of the longitudinal dispersion coefficient $D_{\|}$versus the restitution coefficient, we can observe a small evolution of this coefficient with $e_{2}$. Despite first point of Fig. 16(b), a small decrease in $D_{\|}$with $e_{2}$ can be observed.

During collisions, the kinetic energy of moving particles is controlled by the ability of materials to restore energy. As we have shown in the present section, differences in energy dissipation act directly on particle dispersion. More precisely, previous results shows importance of the restitution coefficient $e_{1}$ on longitudinal dispersion. In fact, during flow of particles, not only energy dissipation but the particle energy itself controls the way particles are dispersed in the porous medium.

\section{E. Effect of particle density $\rho_{s}$}

Finally, to complete our analysis of dispersion energy, we will now consider the influence of $\rho_{s}$ : the density of particles. We have tested how the particle dispersion is affected by the density of the small moving particles $\rho_{s}$. Figure 17 shows the dependency of the two dispersion coefficients, $D_{\|}$and $D_{\perp}$, on $\rho_{s}$. We can notice that the longitudinal dispersion $D_{\|}$increases, while the transverse dispersion $D_{\perp}$ decreases with

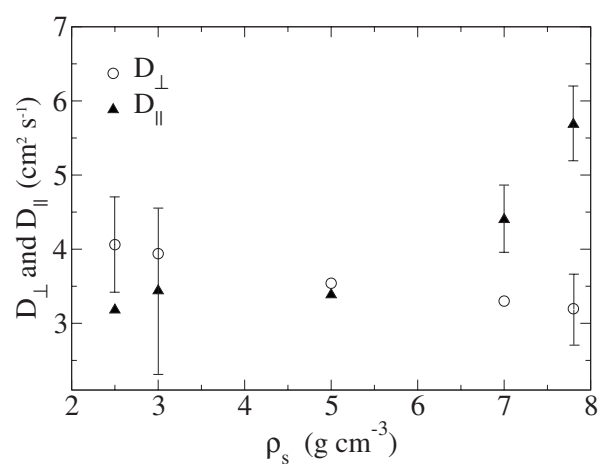

FIG. 17. Evolution of $D_{\|}$and $D_{\perp}$ versus density $\rho_{s}$ of the small particles for $N=2000, D / d=10, D=20 \mathrm{~mm}, H / D=14, e_{2}=0.99$, and $e_{1}=0.84$. 
the increasing of the density of the small moving particles.

For smaller density, gravity force is reduced and the particles can explore laterally the porous medium more easily. On the other side, when the density of moving particles is increased, the gravity force is more important and the distance along the flow direction, between particles that pass a grain layer and ones that do not, is longer. These considerations explain both the decrease in $D_{\perp}$ and the increase in $D_{\|}$ with increasing density of small particles. These results confirm assumptions made in Sec. IV A when the steel beads were replaced with the glass beads. The differences of behavior observed in Fig. 7 for glass or steel beads can be explained by the combination of the two competing effects coming from the density difference in one way and the energy restitution coefficient in the other way. The glass beads have a lower density but higher $e_{2}$ restitution coefficient than steel ones [46]. So, $e_{1}$ is also higher for a collision between two glass particles than for a collision between a glass particle and a steel one. By considering Figs. 15-17, we can clearly understand how $D_{\perp}$ is affected when we replace steel beads with glass ones. The two competing effects of $\rho_{s}$ and energy restitutions give us the explanation about why steel beads have a smaller $D_{\perp}$ than the glass beads, as observed experimentally in Fig. 7.

\section{CONCLUSION}

In this paper, we have performed a large series of experimental and numerical analyses in order to determine the crucial parameters which control the longitudinal $D_{\|}$and transverse $D_{\perp}$ dispersion coefficients in our spontaneous percolation problem. Our experimental setup was modeled as close as possible by our numerical simulations.

Our results show that flow of a particle blob through a packing of larger sphere is a diffusive process. So, we have extended the observations of Ippolito et al. [12] on diffusive behavior to the case where several particles transit at the same time in a porous structure. The first parameter which controls the blob dispersion is the number $N$ of particles. This parameter was the first investigated, and we have experimentally and numerically put in evidence that dispersion coefficients increase with the number of particles flowing simultaneously in the porous medium. With numerical simulations, differences in evolution of dispersion coefficients with $N$ for different size ratio $D / d$ have been observed. This led us to perform investigations on the influence of the parameter $D / d$ on dispersion properties. In a general manner, when size ratio $D / d$ increases, pores crossing are made easier whatever is the considered space direction. This result in observations of an increase in $D_{\perp}$ and a decrease in $D_{\|}$ with $D / d$. Moreover, asymptotic value independent on $D / d$ seems to be reached for size ratio greater than 12 . The parameter $D / d$, combined with $N$ in the parameter $N_{p}$, controls the probability of particles to move freely in the porous space. Dispersion of particles results from collisions with the porous matrix and with other moving particles. It is possible to characterize these collisions with the use of energy restitution coefficient. The first one, whose influence was investigated was $e_{1}$, the restitution coefficient between a moving
TABLE I. Evolution of $D_{\|}$and $D_{\perp}$ versus all studied parameters. In this table, we consider that the parameters in the left column increase. We should notice that an assumption is made for the evolutions of $D_{\|}$and $D_{\perp}$ with $N_{p}$. In fact, our simulations have shown a difference of behavior between cases with $D / d=10$ and ones with $D / d=16$ when studying influence of $N_{p}$ on dispersion coefficients. Regarding results concerning influence of $D / d$ on $D_{\|}$and $D_{\perp}$, which differ around the threshold value of $D / d=12$, we can legitimately assume that this threshold ratio separates the distinct evolutions of dispersion coefficients with $N_{p}$.

\begin{tabular}{|c|c|c|c|c|}
\hline & \multicolumn{2}{|c|}{$D_{\|}$} & \multicolumn{2}{|c|}{$D_{\perp}$} \\
\hline & $D / d<12$ & $D / d>12$ & $D / d<12$ & $D / d>12$ \\
\hline$D / d$ & $\searrow$ & $\longleftrightarrow$ & $\nearrow$ & $\longleftrightarrow$ \\
\hline$N_{p}(N)$ & $\nearrow$ & $\nearrow$ & $\longleftrightarrow$ & $\nearrow$ \\
\hline$e_{1}$ & & & & \\
\hline$e_{2}$ & & & & \\
\hline$\rho_{s}$ & & & & \\
\hline
\end{tabular}

bead and a sphere of the porous medium. Results presented in Sec. VD show that longitudinal dispersion decreases when energy restitution increases, resulting from more difficulties to lost kinetic energy. Our results have also shown that transverse dispersion does not depend on $e_{1}$. This result is very important and illustrates collective effects during the flow of a particle blob. Indeed, Bridgwater et al. [10] showed that the transverse dispersion of an isolated particle increases with this restitution coefficient. Here, the nondependency of $D_{\perp}$ on $e_{1}$ is probably due to some jamming effects which restrict the transverse exploration. Quite similar dependencies were observed for dispersion coefficients with $e_{2}$. Finally, effect of percolating particle density has been investigated. It has been found that $D_{\perp}$ decreases and that $D_{\|}$ increases when this density increases. This can be easily explained by considering changes in magnitude of gravity force. We have experimentally seen that combined influences of parameters can be observed and can sometimes lead to difficulties in physical and practical analyses. Thanks to numerical DEM simulations, it was possible to study influence of parameters separately.

This work deals with a lot of experiments and simulations to presents comparative and exhaustive results. In order to summarize the different parameter evolution analysis, Table I provides the evolutions of the dispersion coefficients when the different studied parameters $\left(D / d, N, e_{1}, e_{2}, \rho_{s}\right)$ increase. In addition to its interest in understanding particle size segregation mechanism in a packing of larger spheres, this work could also be useful to maximize particle diffusion when attempting to realize homogeneous mixtures by gravity driven flows.

\section{ACKNOWLEDGMENTS}

The authors would like to thank S. McNamara for his helpful comments on their work. We are also thankful to the reviewers for their remarks and advice on our work. 
[1] J. C. Williams, Powder Technol. 15, 245 (1976).

[2] J. C. Williams (unpublished).

[3] A. D. Rosato, F. Prinz, K. J. Standburg, and R. Swendsen, Powder Technol. 49, 59 (1986).

[4] A. Rosato, K. J. Strandburg, F. Prinz, and R. H. Swendsen, Phys. Rev. Lett. 58, 1038 (1987).

[5] R. Jullien and P. Meakin, Nature (London) 344, 425 (1990).

[6] G. C. Barker and A. Mehta, Nature (London) 364, 486 (1993).

[7] K. M. Hill and J. Kakalios, Phys. Rev. E 52, 4393 (1995).

[8] J. Duran, T. Mazozi, E. Clément, and J. Rajchenbach, Phys. Rev. E 50, 5138 (1994).

[9] G. Félix and N. Thomas, Phys. Rev. E 70, 051307 (2004).

[10] J. Bridgwater, N. W. Sharpe, and D. C. Stocker, Trans. Inst. Chem. Eng. 47, T114 (1969).

[11] L. Samson, Ph.D. thesis, University of Rennes 1, 1997 (unpublished).

[12] I. Ippolito, L. Samson, and J. P. Hulin, Eur. Phys. J. E 3, 227 (2000).

[13] L. Oger, J. P. Troadec, and B. Tharaud, in World Congress on Particle Technology 4, edited by J. Raper (The Technical Committee, Sydney, 2002), p. 175.

[14] F. Lominé and L. Oger, J. Stat. Mech.: Theory Exp. (2006) P07019.

[15] J. Bridgwater and N. D. Ingram, Trans. Inst. Chem. Eng. 49, 163 (1971).

[16] F. Lominé, Ph.D. thesis, University of Rennes 1, 2007 (unpublished).

[17] L. Oger and F. Lominé, in Powders and Grains, edited by R. Garcia-Rojo, G.-R. Herrmann and S. McNamara (Taylor \& Francis, London, 2005), Vol. 1, pp. 57-61.

[18] W. E. Ranz, Chem. Eng. Prog. 48, 247 (1952).

[19] J. A. Dodds, J. Colloid Interface Sci. 77, 317 (1980).

[20] J. D. Bernal, Nature (London) 183, 141 (1959).

[21] H. J. Frost and R. Raj, J. Am. Ceram. Soc. 65, C19 (1982).

[22] P. Philippe, Ph.D. thesis, University of Rennes 1, 2002 (unpublished).

[23] M. J. Powell, Powder Technol. 25, 45 (1980).

[24] S. Luding, E. Clément, A. Blumen, J. Rajchenbach, and J. Duran, Phys. Rev. E 49, 1634 (1994).
[25] S. McNamara and W. R. Young, Phys. Rev. E 53, 5089 (1996).

[26] S. McNamara and W. R. Young, Phys. Fluids A 4, 496 (1992).

[27] S. McNamara and W. R. Young, Phys. Rev. E 50, R28 (1994).

[28] T. Zhou and L. P. Kadanoff, Phys. Rev. E 54, 623 (1996).

[29] P. A. Cundall and O. D. L. Strack, Geotechnique 29, 47 (1979).

[30] L. Oger, S. Savage, D. Corriveau, and M. Sayed, Mech. Mater. 27, 189 (1998).

[31] K. D. Kafui and C. Thornton, Powder Technol. 109, 113 (2000)

[32] H. Hertz, J. Reine Angew. Math. 92, 156 (1881).

[33] O. R. Walton and R. L. Braun, J. Rheol. 30, 949 (1986).

[34] C. A. Coulomb, Recueil des savants étrangers de l'Académie royale des sciences T.10, 161 (1781).

[35] P. K. Haff and B. T. Werner, Powder Technol. 48, 239 (1986).

[36] O. R. Walton, in Mobile Particulate Systems, edited by E. Guazzelli and L. Oger (Kluwer Academic Publishers, Dordrecht, 1995), pp. 367-379.

[37] R. Gutfraind, O. Pouliquen, and S. Savage, in Engineering Mechanics: Proceedings of the 10th Conference, University of Colorado at Boulder, Boulder, Colorado, 1995, edited by S. Sture (ASCE, 1995), pp. 794-797.

[38] R. Gutfraind and S. B. Savage, J. Geophys. Res. 102, 12647 (1997).

[39] L. E. Silbert, D. Ertas, G. S. Grest, T. C. Halsey, D. Levine, and S. J. Plimpton, Phys. Rev. E 64, 051302 (2001).

[40] L. Verlet, Phys. Rev. 159, 98 (1967).

[41] J. M. Haile, Molecular Dynamics Simulation: Elementary Methods (John Wiley and Sons, New York, 1992).

[42] D. Harleman and R. Rumer, J. Fluid Mech. 16, 385 (1963).

[43] J. Bear, Dynamics of Fluids in Porous Media (Elsevier, New York, 1972).

[44] M. T. Van Genuchten, J. Hydrol. 49, 213 (1981).

[45] M. T. Van Genuchten and W. J. Alves, Agricultural Research Service, U. S. Department of Agriculture Technical Bulletin No. 1661, 1982.

[46] W. Goldsmith, Impact: The Theory and Physical Behavior of Colliding Solids (Edward Arnold Ltd., London, 1960). 